\title{
The response of global seismicity to solar flares of September, 2017
}

\author{
Yu. Ruzhin ${ }^{1}$, V. Novikov ${ }^{2}$ \\ ${ }^{1}$ 1Pushkov Institute of Terrestrial Magnetism, Ionosphere and Radio Wave Propagation of Russian Academy of \\ Sciences, IZMIRAN, Troitsk, Moscow region, Russian Federation, 142190, \\ ruzhin@izmiran.ru \\ ${ }^{2}$ Joint Institute for High Temperatures of Russian Academy of Sciences, 13, bld.2, Izhorskaya str., Moscow, Russian \\ Federation, 125412, \\ novikov@ihed.ras.ru
}

\begin{abstract}
Results of an analysis of interrelation between powerful solar flares of September, 2017 and global seismic activity are presented. It was found that during the period of solar flares and one week after it the number of earthquakes rises by up to $100 \%$. We attribute the obtained results to earthquake triggering effects of solar flares. A possibility of electric/electromagnetic triggering of earthquakes due to space weather disturbances is supported by results of field and laboratory experiments, where earthquakes (both natural and laboratory) were initiated by injection of electrical current into the Earth crust or simulated seismogenic fault. For the case of man-made electric earthquake triggering the current density at the depth of earthquake sources is comparable with estimations of a density of telluric currents induced by variation of space weather conditions due to solar flares.
\end{abstract}

Key words: solar flare, seismic activity, telluric currents, earthquake triggering

\section{Introduction}

To-date quite a large number of studies have been completed to find a correlations between the Earth's seismicity and solar processes. Results of these statistical studies are contradictory: some researchers found the direct correlation between the energy or number of earthquakes and Wolf numbers [Sytinsky, 1987; Sytinsky and Oborin, 1997; Lotsinskaya, 1999], and others - an anti-correlation, [e.g. Sobolev et al., 1998]. According to the study [Sytinsky, 1987] there is a definite dependence of seismicity on the 11year cycle of solar activity, which was verified by the experimental prediction of global seismicity and seismicity of specific regions. The author considered a distribution of the annual average energy of 
earthquakes Ek and the annual numbers Nk of earthquakes of various magnitudes during 11-year cycle of solar activity for 1902-1977 years. It was noted a clear dependence of the Earth seismic activity on the phases of 11-year cycle of solar activity. High seismicity level is observed in the 1st, 3rd, and 6th year (with a probability of 0.99 ) after the maximum of solar activity.

Nevertheless, there are opposite statements as well that the 11-year cycles of seismic activity have a significant negative correlation with cycles of solar activity and geomagnetic disturbances [Sobolev et al., 1998]. Moreover, there is some skepticism in assessing the existence of such solar-terrestrial relations resulted in statistically significant seismicity variations [e.g., Love and Thomas, 2013].

It should be noted that practically all the mentioned conclusions (both optimistic and pessimistic) are based on correlation analysis only. Even when such analysis yields statistically significant results, the authors only assume that there is a possible triggering mechanism in the case of positive correlation, without attempts of understanding the physics of the possible relations, or they provide the most general considerations [Georgieva et al., 2002]. For example, it was noted that earthquakes occur more often when the solar activity level drastically changes. During a solar flare the radiation level increases many times, which interacts with the Earth's magnetosphere resulted in its disturbance and occurrence of magnetic storm. In turn, the magnetic storms may influence to the rotation speed of the Earth, intensity of telluric currents in the lithosphere that results in increase of stresses in the Earth crust [Mazur and Ivanov, 2004; Duma and Ruzhin, 2003]. Some researches [e.g. Riccardi, 2007] connect the variations of seismic activity after solar flares with an increase of a number of cyclones that can act as a trigger due to sharp variations in atmospheric pressure during passage of their fronts over the earthquake source. Nevertheless, the estimations demonstrate that such variations are substantially lower than the level of stress variations due to lunar-solar tides whose influence on seismicity is still under discussion [Beeler and Lockner, 2003].

In the recent years a few papers were published where hypotheses were proposed on the triggering impact of geomagnetic field variations (e.g., during sudden commencement storms) [Sobolev et al., 2001; Zakrzhevskaya and Sobolev, 2002; Tarasov and Tarasova, 2002; Sobolev and Ponomarev, 2003], as well as diurnal Sq-variations [Duma and Ruzhin, 2003]. Particularly, results of statistical analysis obtained for different regions (Northern America, Central Europe, China, and Japan) demonstrated an evident correlation between geomagnetic field variations and a number of earthquakes both for average diurnal cycle and for a long period of about 150 years. An idea was discussed on a possible generation of 
additional stresses in the Earth crust due to geomagnetic field variations [Duma and Ruzhin, 2003]. For clarification of the problem some additional statistical studies are required.

\section{New evidence of earthquake triggering by solar flares}

Powerful surge of solar flash activity early in May 2017 during approaching the minimum of $24^{\text {th }}$ solar cycle was accompanied by significant disturbances of space weather. On September 6, 2017 a group of sun spots AR2673 generated a large solar flare of X9.3 class (Fig.1), the strongest flare over the past twelve years. Its explosion produced a coronal mass ejection partially directed towards the Earth. We carried out a statistical analysis of the catalogs of earthquakes USGS and EMSC for determination of effect of solar flares on global seismic activity.
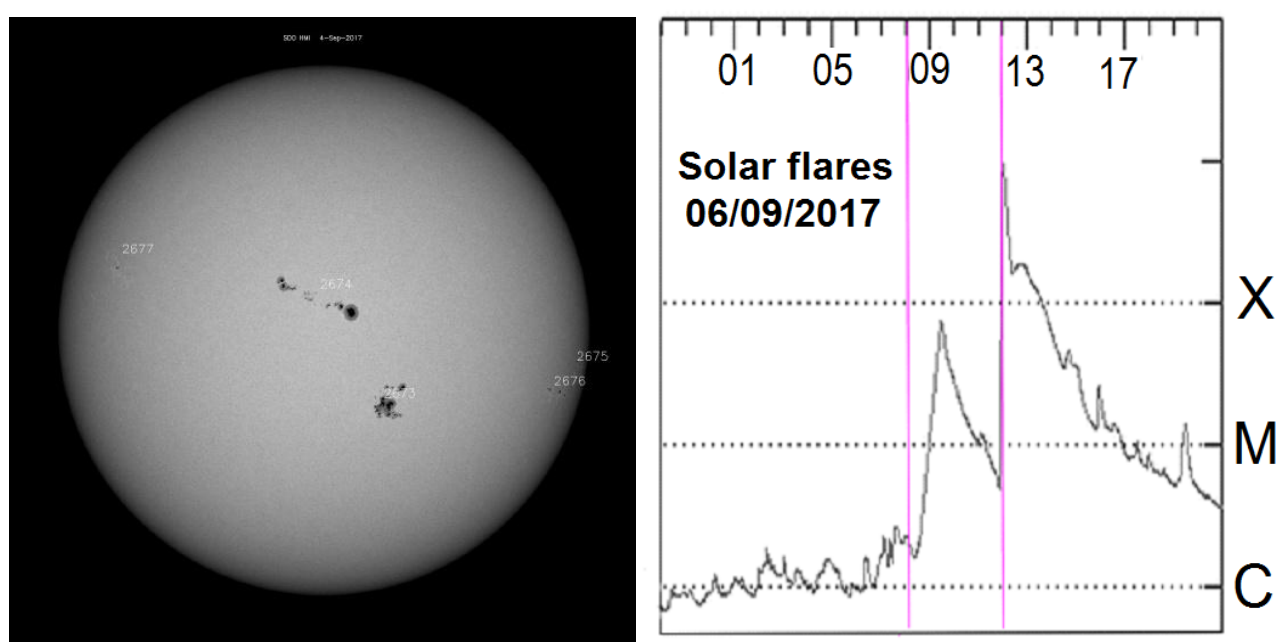

Fig.1. September 6, 2017 marked by two powerful flares of class X (sun spots group AR2673 - see left side). The first flash is X2.2, the maximum at 09:10 UTC (see right side). Flash X9.3, followed three hours later - this is the most powerful in the last 12 years. 


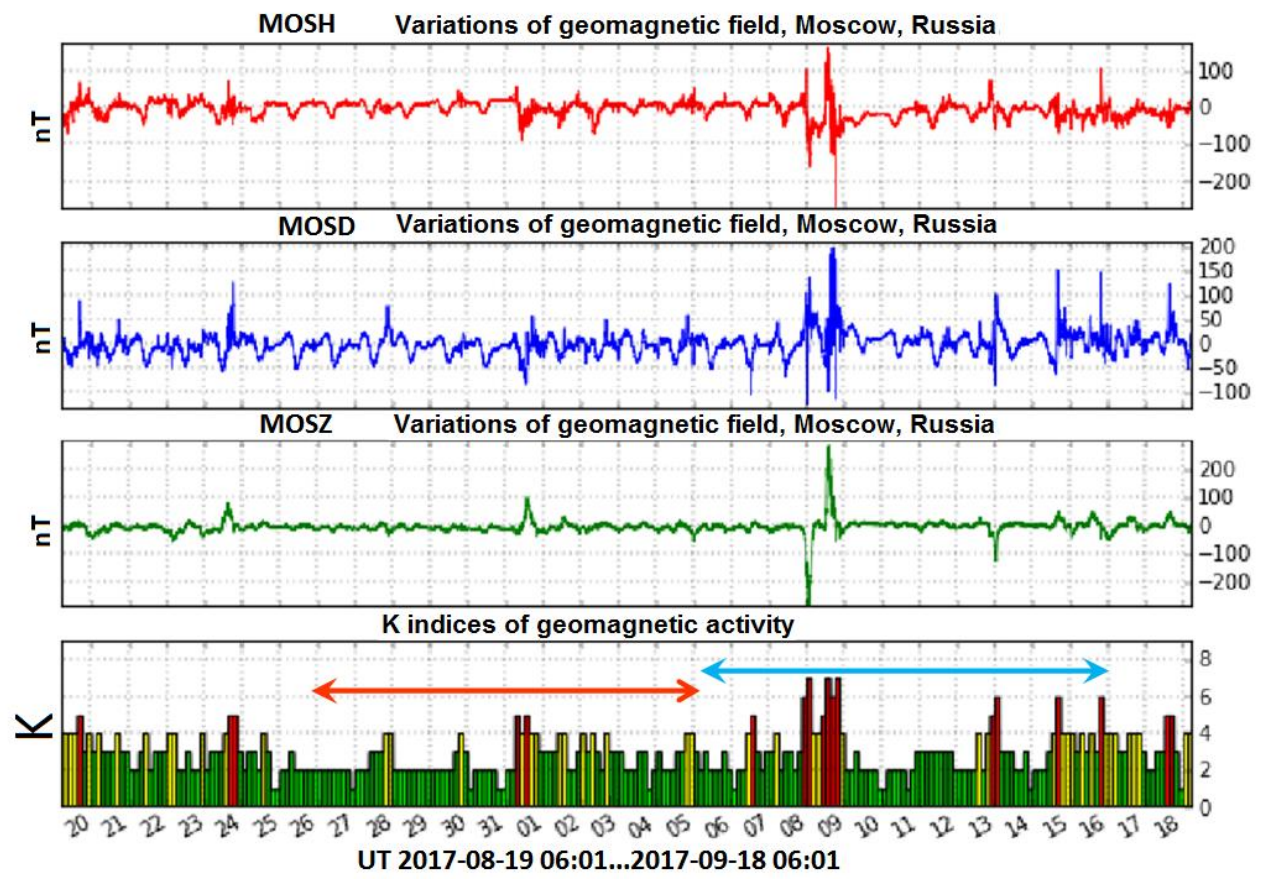

Figure 2. Magnetic activity during a month from August 20, 2017. Arrows designate periods selected for an analysis.

Considering the results published in [Sobolev et al., 2001; Zakrzhevskaya et al., 2002] indicated that the seismic response appeared during the followed 1-6 days, we choose two periods of time: one with flares of a class X (flares on September 6, 7, and 10, 2017), that is, from September 6 to 16 (blue arrow in Figure 2), and the similar background interval one day before, or from August 26 to September 5 (red arrow), inclusive.

Figure 2 shows a magnetogram, i.e. geomagnetic field variations (three components) according to data collected in IZMIRAN observatory during a month (2017.08.20 to 2017.09.20) with the time interval of an analysis denoted by horizontal arrows. The lower part of the graph presents local index $\mathrm{K}$ of magnetic activity. 


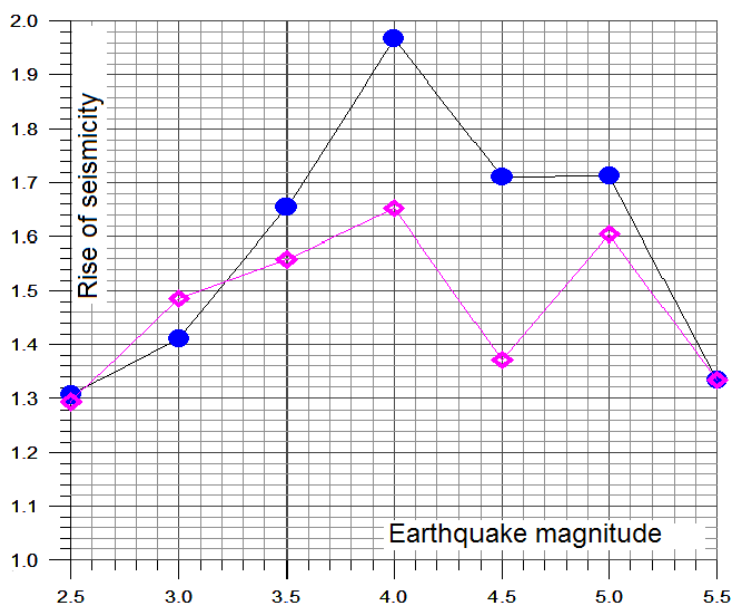

Figure 3. Comparison of earthquake (EQ) number for the similar period (11 days) before and after solar flare. Significant rise of EQ number is observed (up to 100\%) after the flare for EQ magnitude of 2.5 to 5.5. The numerical data are given in Table 1.

Table 1:

\begin{tabular}{|c|c|c|c|}
\hline $\begin{array}{c}\text { Earthquake } \\
\text { magnitude }\end{array}$ & $\begin{array}{c}26.08 .2017- \\
05.09 .2017\end{array}$ & $\begin{array}{c}06.09 .2017- \\
16.09 .2017\end{array}$ & $\begin{array}{c}\text { Relative rise of } \\
\text { earthquake number }\end{array}$ \\
\hline 2.5 & 917 & 1191 & 1.299 \\
\hline 3.0 & 608 & 848 & 1.410 \\
\hline 4.0 & 253 & 498 & 1.968 \\
\hline 5.0 & 35 & 60 & 1.714 \\
\hline 5.5 & 5 & 12 & 1.333 \\
\hline
\end{tabular}

Figure 3 demonstrates a rise of seismic activity according to two earthquake catalogs: USGS [https://earthquake.usgs.gov/earthquakes/search/] and EMSC (https://www.emsc-csem.org/Earthquake/) squares and circles, respectively. A comparison of earthquake number during the same period (11 days) before and after the solar flare demonstrates a significant rise (up to 100\%, blue circles, Fig.3) of a number of earthquakes with a magnitude of 2.5 to 5.5. In so doing, based on these evident results we may state that the solar flares can provoke the earthquake occurrence. One of the possible reasons of earthquake triggering is the sharp increase of telluric currents induced in the conductive areas of geological faults matured for earthquake occurrence [Han et al., 2004]. It should be noted that to-date there are many cases of earthquake triggering by electric current observed under field and laboratory conditions, which we briefly overview below. 


\section{Field and laboratory results on electric triggering of earthquakes}

In the middle of $90^{\text {th }}$ a statistically significant impact of electrical pulses of DC current injected through emitting dipole into the earth crust on the spatial and temporal distribution of weak seismicity of Pamir [Tarasov, 1997] and Northern Tien Shan regions has been discovered [Tarasov et al., 1999, 2000; Tarasov and Tarasova, 2004; Chelidze et al., 2006]. The grounded electrical dipole of about $4 \mathrm{~km}$ length was supplied by single pulses of electrical current of $1.5-2.5 \mathrm{kA}$, voltage of $1 \mathrm{kV}$, and duration of $2.5-10.0$ s provided by geophysical magneto-hydrodynamic (MHD) generator used for deep electromagnetic sounding of the Earth crust for monitoring of the crust stress state and search of electromagnetic precursors of earthquakes. Statistically, by the epoch superposition method with application of data of regional seismic catalogs and catalogs of firing runs of MHD generator it was found that after injection of electric current into the earth crust the number of weak earthquakes $\left(M_{b}<3.0\right)$ is increased by several times with a time delay of 1-2 days, and then within 5-7 days it returns to the previous background level. A hypothesis on triggering action of electrical pulses has been proposed [Zeigarnik et al., 1999]. Laboratory experiments carried out to date with application of various press equipment and samples of geomaterials under stress-strain state demonstrated a modulation of acoustic emission by electric current pulses applied to the sample [Sobolev and Ponomarev, 2003; Bogomolov et al., 2004; Avagimov et al., 2006]. It was shown that a response of acoustic emission to electric action characterized crack formations in the sample rises as the sample loading and maximally appears at the stress level of 0.95-0.99 of breaking stress of the material [Sobolev and Ponomarev, 2003]. The effect of modulation of geoacoustic emission by external actions is well-known, e.g. under impact of lunar-solar earth tides [Gordeev et al., 1995]. Nevertheless, a response of acoustic emission on the external action does not mean that it can unambiguously result in triggering the macro event (earthquake or failure of tested rock sample) [Beeler and Lockner, 2003]. The laboratory study of electric triggering of macro events (laboratory "earthquakes") were carried out at simplified slider system [Chelidze et al., 2002] and spring-block model [Novikov et al., 2017] simulated the seismogenic fault. The both experimental facilities are based on an idea that "stick-slip" behavior of the fault is a mechanism of earthquake preparation and occurrence [Brace and Byerlee, 1966]. In the both series of experiments the sudden slip of the movable block was initiated by injection of electrical current into a contact between the fixed and movable blocks (simulated crust fault). The triggering of slip of movable block of the spring-block model is possible only in the 
subcritical state of the system, when the accumulated shear stresses are $0.98-0.99$ of critical value, when the sharp slip (laboratory "earthquake") occurs [Novikov et al., 2017].

We have to note that the DC current density $\left(10^{-7}-10^{-8} \mathrm{~A} / \mathrm{m}^{2}\right)$ calculated for field conditions of the Bishkek test site during experiments on DC current injection into the Earthquake crust [Novikov et al., 2009] is comparable with the density of telluric currents induced in the Earth crust due to solar flares [Sorokin et al., 2012]. Based on the results described above we can state that the earthquake triggering due to solar flares is possible. Keeping in mind that the threshold current density triggered the seismic event under laboratory conditions is about $20 \mathrm{~A} / \mathrm{m}^{2}$ [Novikov et al., 2017] that exceeds by several orders the current density calculated for field experiments carried out at the Bishkek test site, the effect of seismicity response to electrical action may be explained either by electric current contraction in the narrow highly conductive fault zones [Han et al., 2004] or by secondary triggering mechanisms driven by electric pulses, like electromagnetic stimulation of fluid migration into the fault zone resulted in reduction of the fault strength properties [Novikov and Novikova, 2014]. It should be noted that these hypotheses have only a phenomenological character without a detailed theoretical justification and experimental verification. New fundamental knowledge is needed on the mechanisms of interaction of the electromagnetic field with rocks under the stress-strain state, as well as on the influence of electric/electromagnetic fields in combination with natural geophysical fields on deformation processes in the fault with specific parameters (electrical conductivity, orientation and maturity for earthquake occurrence.

\section{Conclusion:}

The overview of studies completed to-date on a relation of the Earth's seismicity and solar processes demonstrates the fuzzy and contradictory results. Nevertheless, by simple statistical analysis of behavior of Earth's seismicity after a large solar flare of X9.3 class on September 6, 2017 (the strongest flare over the past twelve years) we have discovered a new evidence of earthquake triggering. The global number of earthquakes with magnitude of 2.5 to 5.5 within 11 days after the solar flare has increased by 30 to $100 \%$. The possibility of electric/electromagnetic triggering of earthquake due to space weather disturbances is supported by results of field and laboratory studies, where the earthquakes (both natural and laboratory) were initiated by injection of electrical current into the Earth crust keeping in mind that the current 
density at the depth of earthquake sources is comparable with estimations of the density of telluric currents induced by variation of space weather conditions due to solar flares.

\section{Acknowledgments}

The work was supported by RFBR grant No. 18-05-00255.

\section{Reference}

Avagimov A. A., Zeigarnik V.A., Klyuchkin V.N., On the structure of acoustic emission of model samples in response to an external energy action. Phys Solid Earth, 42(10), 824-829, 2006.

Beeler N. M., Lockner D. A., Why earthquakes correlate weakly with the solid Earth tides: Effects of periodic stress on the rate and probability of earthquake occurrence, J. Geophys. Res., 108(B8), 2391, 2003.

Bogomolov L.M., Il'ichev P.V., Novikov V.A., Okunev V.I., Sychev V. N., Zakupin A.S., Acoustic emissions response of rocks to electric power action as seismic-electric effect manifestation, Ann. Geophys., 47(1), 65-72, 2004.

Bogomolov L.M., On a mechanism of electromagnetic effect on kinetics of microfractures and electrically stimulated variations of acoustic emission of rock samples, Phys. Mesomech. 13(3), 39-56, 2010.

Boteler D. H., Pirjola R. J., Nevanlinna H., The effects of geomagnetic disturbances on electrical systems at the Earth's surface, Adv. Space Res., 22(1), 17-27, 1998.

Brace W.F., Byerlee J.D., Stick-slip as a mechanism for earthquakes. Sci. New Series 153(3739), 990992, 1966.

Chelidze T., Varamashvili N., Devidze M., Chelidze Z., Chikladze V., and Matcharashvili T., Laboratory study of electromagnetic initiation of slip, Ann. Geoph., 45 (5), 587-598, 2002.

Chelidze T., De Rubeis V., Matcharshvili, and Tosi P., Influence of strong electromagnetic discharges on the dynamic of earthquakes time distribution in the Bishkek test area (Central Asia), Ann. Geoph., 49(45), 961-975, 2006.

Duma G., Ruzhin Yu., Diurnal changes of earthquake activity and geomagnetic Sq variations, Natural Hazards and Earth System Sciences, 3,171-177, 2003.

EMSC earthquake catalogue - https://www.emsc-csem.org/Earthquake/ 
Georgieva K., Kirov B., Atanasov D., On the relation between solar activity and seismicity on different time scales. J. Atmosph. Electr., 22(3), 291-300, 2002.

Gomberg J., Beeler N.M., Blanpied M.L., Earthquake triggering by static and dynamic deformations. J Geophys Res 103, 24411-24426, 1998.

Gordeev E.I., Saltykov V.A., Synitsyn V.I., Chebrov V.N., Correlation of high-frequency noise with lunar-solar tides. Dokl Earth Sci., 343A (6), 42-45, 1996.

Han Y., Guo Z., Wu J., Ma L., Possible triggering of solar activity to big earthquakes (Ms $\geq 8$ ) in faults with near west-east strike in China, Sci. China Ser. B: Phys. Mech. Astron., 47, 173-181, 2004.

Lanzerotti L. J., Gregori G.P., Telluric currents: The natural environment and interaction with man-made systems, in The Earth's Electrical Environment, Natl. Acad. Press, Washington, D.C., 232-257, 1986.

Lotsinskaya, N. I., Relation of the Earthquake Global Energy to Solar Activity, Vestn. Kiev. Univ., Ser. Astron., 35, 45-50, 1999.

Love J. J., and Thomas J. N., Insignificant solar-terrestrial triggering of earthquakes, Geophys. Res. Lett., 40, 1165-1170, 2013.

Mazur I.I., Ivanov O.P., Dangerous natural processes. Moscow, (in Russian), 2004.

Novikov V.A., Okunev V.I., Klyuchkin V.N., Liu J., Ruzhin Yu.Ya., Shen X., Electrical triggering of earthquakes: Results of laboratory experiments at spring-block models, Earthq. Sci., 30 (4), 167-172, 2017

Novikov V.A., Novikova E.O., Electromagnetic stimulation of fluid migration into fault area and earthquake triggering phenomena, Geophys. Res. Abstr., 16, EGU2014-12790, 2014.

Novikov V., Zeigarnik V., Konev Yu., Rickman V., Electric Triggering of Crack Formations in the Fault for Release of Tectonic Stresses, Eos Trans. AGU, 90(52), Fall Meet. Suppl., Abstract S51C-1447, 2009.

Riccardi U., Hinderer J., and Boy J.-P., On the efficiency of barometric arrays to improve the corrections of atmospheric effects on gravity data, Phys. Earth Planet. Int., 161, 224-242, 2007.

Simpson I.F., Solar activity as a triggering mechanism for earthquakes, Earth and Planet. Sci. Letter., 3(5), 417-425, 1968.

Sobolev G. A., Shestopalov I.P., and Kharin E. P., Implications of Solar Flares for the Seismic Activity of the Earth, Izvestiya, Phys. Solid Earth, 34 (7), 603-607, 1998.

Sobolev G.A., Zakrzhevskaya N.A., and Kharin E.P., On the coupling of seismicity with magnetic storms, Physics of the Solid Earth, 11:62-72, 2001. 
Sobolev G.A., Ponomarev A.V., Physics of earthquakes and precursors, Moscow, Nauka, 2003.

Sorokin V.M., Yaschenko A.K, Ruzhin Yu. Ya., Novikov V.A., Model for solar flare influence to the seismic activity, Proc. EMSEV-2012 Workshop. Gotemba, Japan. October 1-4, 2012. Abstr. 3-10p, 2012.

Sytinsky A.D., Relationship of Earth's seismicity with solar activity and atmospheric processes. Leningrad (in Russian), 1987.

Sytinsky A.D., Oborin D.A., Influence of perturbations of interplanetary medium on seismicity and atmosphere of the Earth, Geomagn. Aeron., 37(2), 138-141, 1997.

Tarasov N.T., Crustal seismicity variation under electric action. Dokl Acad Nauk SSSR, 353(4), 542-545, 1997.

Tarasov N.T., Tarasova N.V., The effect of geomagnetic storms on the seismicity, Proc. of the 3rd International Workshop on Magnetic, Electric and Electromagnetic Methods in Seismology and Volcanology, Moscow, Russia, 3-6 September 2002, 206-208, 2002.

Tarasov N.T., Tarasova N.V., Zeigarnik VA, Avagimov AA, The effect of high energy electromagnetic pulses on seismicity in Central Asia and Kazakhstan, J. Volcanol. Seismol., 21(4-5), 627-639, 2000.

Tarasov N.T. and Tarasova N.V., Spatial-temporal structure of seismicity of the North Tien Shan and its change under effect of high energy electromagnetic pulses, Ann. Geoph., 47(1), 199-212, 2004.

USGS earthquake catalogue - https://earthquake.usgs.gov/earthquakes/search/

Zakrzhevskaya N.A., Sobolev G.A., On the seismicity effect of magnetic storms, Izvestiya, Phys. Solid Earth, 38(4), 249-261, 2002.

Zeigarnik V.A., Avagimov A.A., Tarasov N.T., Managing earthquakes? Science in Russia, 2, 16-21, 1999. 